\title{
Automatic ASN.1 constraint generation for testing purposes
}

\author{
Erik Kwast (e-mail: E.Kwast@research.ptt.nl) \\ PTT Research, Neher Laboratories, \\ P.O. Box 421, 2260 AK Leidschendam, the Netherlands
}

\begin{abstract}
The main task in deriving a TTCN test suite consists of specifying the dynamic behaviour part and the constraints part. The constraints part describes the constraints, i.e. data unit instantiations that are used in the test cases. This paper presents a method to automatically generate such constraints. The method is based on Attributed ASN.I, an extended version of ASN.1 that is used to formalise the relevant data aspects, and linear programming techniques.
\end{abstract}

Keyword Codes: B.4.5, C.2.2, F.4.2

Keywords: Reliability, Testing and Fault Tolerance, Network Protocols, Grammars and Other Rewriting Systems.

\section{Introduction}

ASN.1 is a description language that is used in protocol standards to define the data unit ${ }^{1}$ types used by these protocols. Furthermore, ASN.1 and its associated value notation are used in TTCN [5, part 3] test suites to specify constraints, i.e. data unit instantiations that are used in the test cases of the dynamic behaviour part. The derivation of constraints is a complex task as different restrictions apply to the values that may be assigned to the fields of a data unit. Apart from regular value domain restrictions, the restrictions are due to the dependencies that exist between the fields of the data units. The following dependencies can be identified.

External dependencies: the presence or absence of optional data unit fields, or the specific value that can be assigned to a field of a specific data unit in a communication sequence may rely on the presence, absence or value of a field of a data unit that was previously exchanged.

Internal dependencies: the presence or absence of optional data unit fields, or the specific value that can be assigned to a data unit field may rely on the presence, absence or value of another field of that same data unit. 


\section{[5] D_ABORT_REQ ::= [APPLICATION 13] IMPLICIT SEQUENCE $\{\ldots$ \\ aBORTReason [1] INTEGER $\{\ldots$, invalid-parameter $(1), \ldots\}$, Reflected-parameter [2] IMPLICIT BIT STRING OPTIONAL, -8 bits maximum, only if abortReason is invalid parameter $\ldots\}$}

Figure 1: Example of an internal dependency

In this paper, we describe a solution to the constraint generation problem with respect to internal dependencies. This solution is based on Attributed ASN.1 [3] and linear programming techniques [7]. Ideas to automatically generate ASN.1 constraints, taking the external dependencies into account, have been presented in [6]. These two partial solutions to generate constraints can be combined into a method to automatically generate ASN.1 constraints that conform to both internal and external dependencies.

The relevance of internal dependencies, of which figure 1 shows a typical example taken from [4], follows from [3, 8]. These dependencies can be formalised by using Attributed ASN.1, which is an extension of ASN.1 based on the theory of attribute grammars. The syntactic structure of Attributed ASN.1 types is described by ASN.1. Additionally, it is possible to associate a set of attributes with any field of the ASN.1 type description. There are two kinds of attributes, i.e., predefined attributes and user defined attributes. The predefined attributes are always implicitly defined. Examples of predefined attributes are: present denoting the presence or absence of OPTIONAL fields and value denoting the value of an ASN.1 Simple Type. The user defined attributes have to be declared and defined explicitly. The declaration of user defined attributes consists of the declaration of the name and the type of this attribute. The definition of a user defined attribute is done by specifying a rule, which describes how its value is computed using values of other user defined and predefined attributes.

The relations between attributes are specified by predicates that are called conditions. The values defined by an Attributed ASN.1 specifications are those values defined by the underlying ASN.1 specification for which all rules and conditions are satisfied. The internal dependency presented in figure 1 is for example formalised by the following conditions:

IF aBortReason.value $\neq$ invalid-parameter THEN reflected-parameter.present = FALSE ELSE TRUE FI;

reflected-parameter.length $\leq 8$;

The structure of the rest of this paper is as follows. Section 2 presents an overview of our solution to generate constraints based on Attributed ASN.1 and linear programming techniques. Section 3 presents a worked out example of the generation of a constraint. Finally, section 4 describes some issues regarding the practical feasibility of the approach as well as some conclusions.

\footnotetext{
${ }^{1}$ With the term data unit we refer to both Protocol Data Units and Abstract Service Primitives.
} 


\section{Constraint generation from attributed ASN.1}

In this section we present an overview of our approach to generate constraints from Attributed ASN.1 specifications. This approach consists of the following four steps:

Step 1: $\{$ List all relevant restrictions $\}$

To construct a constraint for a specific type $\mathrm{T}$, we recursively list all rules and conditions of $\mathrm{T}$ and its subtypes. This list thus contains all restrictions that shall be satisfied by the constraint to be constructed.

\section{Step 2: \{Transform the list of restrictions into a linear problem specification\}}

Attributed ASN.1 does not put any restrictions on the form that the expressions in the rules and condition may take. One may for example use undecidable predicates as conditions. For generation purposes, we evidently have to limit the expressive power of Attributed ASN.1. Given the fact that we want to use linear programming techniques we (only) require that rules and conditions shall only use linear expressions. From this requirement and the fact that all predefined attributes are (representable by) either integers or booleans, it easily follows that the value of any attribute can be represented by an integer, a real or a boolean. So, the list of rules and conditions obtained in step 1 can be transform into a linear problem specification. It should be noted that this requirement seems to be no problem when using Attributed ASN.1 to specify data types for practical protocols.

\begin{tabular}{|l|c|c|c|}
\hline original predicate & \multicolumn{3}{|c|}{ offsprings } \\
\hline P AND Q & $\begin{array}{l}\mathrm{P} \\
\mathrm{Q}\end{array}$ & $\neg \mathrm{P}$ & $\neg \mathrm{Q}$ \\
\hline $\mathrm{P}$ OR Q & $\mathrm{P}$ & $\mathrm{Q}$ & $\neg \mathrm{P}$ \\
& & & $\neg \mathrm{Q}$ \\
\hline $\begin{array}{l}\text { IF guard THEN e1 } \\
\text { ELSE e2 FI }\end{array}$ & $\begin{array}{c}\text { guard } \\
\mathrm{e} 1\end{array}$ & $\begin{array}{c}\neg \text { guard } \\
\mathrm{e} 2\end{array}$ & \\
\hline $\mathrm{x}=$ IF guard \\
THEN e1 ELSE e2 FI & $\begin{array}{c}\text { guard } \\
\mathrm{x}=\mathrm{e}\end{array}$ & $\begin{array}{c}\neg \text { guard } \\
\mathrm{x}=\mathrm{e} 2\end{array}$ & \\
\hline $\mathrm{x}=\mathrm{P}$ & $\mathrm{x}=$ TRUE \\
& $\mathrm{P}=$ FALSE & \\
\hline $\mathrm{y} \neq \mathrm{z}$ & $\mathrm{y}<\mathrm{z}$ & $\mathrm{y}>\mathrm{z}$ & \\
\hline
\end{tabular}

Table 1: Predicates and their offsprings

The transformation is done by recursively eliminating all IF/THEN/ELSE expressions and boolean valued operands The boolean values TRUE and FALSE are simply translated into 1 and 0 respectively. The elimination of boolean valued operands and IF/THEN/ELSE expressions is more complex. The reason for this is that this elimination produces several offsprings which should all be taken into account in order to compute a constraint.

The offsprings of the relevant predicates are presented in table 1 , in which $P$ and $Q$ represent predicates, $x$ represents a boolean valued attribute and $y$ and $z$ represent integer or real valued attributes. 
Step 3: \{Solve the linear problem using linear programming techniques [7]\}

The result of step 2 consists of a set of linear problem specifications which should all be considered to generate a constraint. As all attributes in these linear problem specifications are either integers or reals, we can use mixed integer linear programming techniques to compute solutions. These techniques are designed to find optimum solution given a list of linear equations and a function to be optimised. If we are just interested to find $a$ solution, we can use a constant function as the optimum function. However, it is also possible to specify that for example a constraint with minimum length should be generated.

Some of the linear problem specifications resulting from step 2 may be infeasible as the transformation may produce a problem specifications with contradicting equations. In practice, this does not pose any problems as linear programming techniques are capable of detecting infeasible problems. It is, however, interesting to note that the Attributed ASN.1 specification defines an empty value type set if and only if all the linear problem specifications will be infeasible.

Step 4: \{Construct the constraint from the computed solution\}

Constructing a constraint means that values must be assigned to all ASN.1 fields of type Simple Type, unless it is a field in an absent optional subtype. All necessary information to do so is provided by the solution(s) of step 3. It is possible that some predefined value attributes are not restricted in a computed solution. For the fields associated with these attributes, we can simply use a random value taken from the relevant domain to construct the constraint if the associated data unit is sent by the tester. In case the associated data unit is received by the tester we can use the constraint wild card symbols, i.e., "*” and "?".

\section{Constraint generation example}

In this section we present an example of the constraint generation approach described above. This will be done by constructing a constraint of type T2 as defined in figure 2. Type T2 is a simulation of a SEQUENCE OF T1 with at least one T1. This somewhat artificial example is chosen as it nicely shows all the problems that one may encounter in generating constraints. Furthermore, it nicely shows the expressive power of Attributed ASN.1, even if only linear expressions are allowed.

The basic ASN.1 structure for T1 is one boolean and two integers. T1 has two user defined attributes (also called properties). The first being T1.max which is a synthesized attribute that takes the value of the maximum of the two integers and T1.extbool which is an inherited attribute that is set by type T2. The attribute T1.max is not used in the type T1 itself, however it is used in the type T2 (referred to by f1.max). The basic ASN.1 structure for T2 is one T1 and one optional T2. T2 has one user defined attribute, i.e., T2.max which takes the value of $\mathrm{f} 1$.max and hence the maximum value of the two integers of $\mathrm{f} 1$. This example also shows the use of two kinds of predefined attributes. The first one holds the value of an ASN.1 Simple Type field, e.g., i1.value, The second one, indicates the absence or presence of an optional field, e.g., f2.present.

The constraint, denoted by $M$, that we will construct is a specific instantiation of type $T 2$. From the type definition $\mathrm{T} 2$ of $\mathrm{M}$ it follows that we have to construct an instantiation of $\mathrm{T} 1$, denoted by M.f1 and possibly another T2, denoted by M.f2. As will be clear, the problem of 


$$
\begin{array}{r}
\text { T1 ::= PROPERTIES max : INT, } \\
\text { extbool : BOOL }
\end{array}
$$

SEQUENCE \{

b BOOLEAN,

i1 INTEGER,

i2 INTEGER\}

RULES:

$\max :=$ IF i1.value $>$ i 2 .value

THEN il.value

ELSE i2.value FI;

CONDITIONS:

IF b.value AND extbool

THEN i1.value $>$ i2.value

ELSE TRUE FI;
T2 ::= PROPERTIES max : INT

SEQUENCE\{

f1 T1,

f2 T2 OPTIONAL\}

RULES:

$\max :=\mathrm{f} 1 . \max ;$

f1.extbool := IF f2.present

THEN f1.max $>(2 *$ f2.max $)$

ELSE FALSE FI;

CONDITIONS:

IF f2.present

THEN f1.max $>$ f 2 .max ELSE TRUE FI;

IF f2.present

THEN TRUE ELSE T2.max $>0 \mathrm{FI}$;

Figure 2: Attributed ASN.1 specification for constraint generation example

the recursive construction of instantiations of type $T 2$ in the construction of $M$ has to be solved first. To prevent the example from becoming to long, we chose a recursive depth of 1 for $\mathrm{M}$. So, $\mathrm{M}$ incorporates an instantiation of type $\mathrm{T} 2$, which itself does not contain an instantiation of type T2. Using attributes, this can be expressed more formally as: M.f2.present $=$ TRUE AND M.f2.f2.present = FALSE. Having decided upon the recursive construction problem, we can start the construction of $\mathrm{M}$.

Step 1: Listing all relevant rules and conditions for $M$ results in the list presented in figure 3. This list contains all conditions and rules that follow from the Attributed ASN.1 definitions of type T2. It should be noted, however, that we have replaced the attribute names as they occur in the type definition by the names of the attributes as they are for the specific instantiation $\mathrm{M}$. This makes the attributes uniquely identifiable which is necessary to derive the proper problem specification. Furthermore, the restrictions that were added to stop the recursive construction of instantiations of type T2 have been added. It should be noted that the rules which originally were assignments, or rather definitions of attributes, changed into restrictive predicates.

Step 2: The list that is constructed in step 1 is transformed into a set of linear problem specifications by eliminating all boolean valued operators and attributes. One specific linear problem from this set is presented in figure 4 . Every equation in this figure is preceded by a number indicating its parent equation from figure 3 . Furthermore, the $*$ symbol is used to indicated cases that one of several possible offsprings was chosen for our example.

Step 3: A solution to the problem presented in figure 4 defines a conforming instantiation of type T2. Such a solution can be computed by using linear programming techniques, if an optimisation function is specified. If we are for example interested in an instantiation of T2 with a maximum value for M.f1.il we can use M.fl.il.value as the target function. In this case we evidently have to specify an upper limit for the domain of M.f1.i1. Using a constant target function, a linear programming tool may compute the solution of figure 5 . 

\{Top level T2: $M$ \}
(1) M.max = M.f1.max;
(2) M.f1.extbool = IF M.f2.present THEN M.f1.max > (2* M.f2.max)
ELSE FALSE FI;
(3) IF M.f2.present THEN M.f1.max > M.f2.max ELSE TRUE FI;
(4) IF M.f2.present THEN TRUE ELSE M.max > 0 FI;
\{First level T1: M.f1\}
(5) M.f1.max = IF M.f1.i1.value > M.f1.i2.value THEN M.f1.i1.value ELSE M.f1.i2.value FI;
(6) IF M.f1.b.value AND M.f1.extbool THEN M.f1.i1.value > M.f1.i2.value

\{First level T2: M.f2\}

(7) M.f2.max = M.f2.f1.max;

(8) M.f2.f1.extbool = IF M.f2.f2.present THEN M.f2.f1 $\max >(2 *$ M.f2.f2.max $)$ ELSE FALSE FI;

(9) IF M.f2.f2.present THEN M.f2.f1.max > M.f2.f2.max ELSE TRUE FI;

(10) IF M.f2.f2.present THEN TRUE ELSE M.f2.max > 0 FI;

\{Second level T1: M.f2.f1\}

(11) M.f2.f1.max = IF M.f2.f1.i1.value > M.f2.f1.i2.value THEN M.f2.f1.i1.value

(12)

(12) IF M.f2.f1.b.value AND M.f2.f1.extbool THEN M.f2.f1.i1.value > M.f2.f1.i2.value ELSE TRUE FI;

\{Additional restrictions\}

(13) M.f2.present = TRUE;

(14) M.f2.f2.present = FALSE;

Figure 3: List of restrictions for constraint $\mathrm{M}$ of type $\mathrm{T} 2$
(1) M.max = M.f1.max;
(2*) M.f2.present $=1$;
(2*) M.f1.extbool = 1;
(2) M.f1.max $>(2 *$ M.f2.max);
(3*) M.f2.present $=1$;
(3) M.f1.max > M.f2.max;
(4*) M.f2.present = 1;
(5*) M.f1.i1.value > M.f1.i2.value;
(5) M.f1.max = M.f1.il.value;
(6*) M.fl.b.value = 1;
$\left(6^{*}\right) \quad$ M.f1.extbool = 1;
(6) M.f1.i1.value > M.f1.i2.value;
(7) $\quad$ M.f2.max $=$ M.f2.f1.max;
$\left(8^{*}\right) \quad$ M.f2.f2.present $=0$;
(8) $\quad$ M.f2.f1.extbool =0;
$\left(9^{*}\right) \quad$ M.f2.f2.present $=0$;
(10*) M.f2.f2.present $=0$;
(10) M.f2.max > 0;
$\left(11^{*}\right)$ M.f2.f1.i1.value $\leq$ M.f2.f1.i2.value;
(11) M.f2.f1.max = M.f2.f1.i2.value;
(12*) M.f2.f1.extbool = 0;
(13) M.f2.present = 1;
(14) M.f2.f2.present $=0$;

Figure 4: Linear problem specification for constraint $M$ 


\begin{tabular}{|llll|}
\hline M.f1.b.value & $=1$ & M.f2.f1.i1.value $=1$ \\
M.f1.extbool $=1$ & M.f2.f1.i2.value $=1$ \\
M.f1.i1.value $=3$ & M.f2.f1.max & $=1$ \\
M.f1.i2.value $=0$ & M.f2.f2.present $=0$ \\
M.f1.max & $=3$ & M.f2.max & $=1$ \\
M.f2.f1.extbool $=0$ & M.f2.present & $=1$ \\
& M.max & $=3$
\end{tabular}

Figure 5: Linear problem solution of the example

Step 4: Constraint $M$ can be easily constructed from the computed solution. It should be noted that the solution of figure 5 does not restrict M.f2.f1.b.value which thus can be randomly set to, e.g., 0 . Doing so results in $M=\{\{$ TRUE, 3,0$\},\{$ FALSE, 1,1$\}\}\}$

\section{Practical feasibility and conclusion}

To show the practical feasibility of our approach we have implemented our constraint generation method in a prototype tool. We found that the performance and capabilities of the tool mainly depends on the following two aspects:

- the performance and capabilities of the linear programming software.

- the number of linear program specifications that have to be considered to compute a constraint for a specific Attributed ASN.1 type.

Regarding these aspects we have the following experiences. The linear programming tool ${ }^{2}$ we used, implements a branch and bound technique to solve mixed integer linear programming problems. It is said to solve integer linear programming problems with about 100 variables in reasonable time, provided that the problem is not too complex. The constraint generation tests that we performed, however, did not even come close to this limit. The order of the computation time we experienced is seconds rather then minutes.

The number of linear programming problems that have to be considered to compute a constraint grows exponentially with the number of boolean operators and attributes. However, many of these transformed problem specifications are infeasible. Most of the time the infeasibility is easily (and quickly) detected, as the problems contain equations of the form $x=0 ; x=1$ which result from the boolean elimination in the second step of our approach. Our overall experience is that the performance of the constraint generation tool is acceptable.

In this paper we have described a method that can be used to automatically generate ASN.1 constraints that conform to internal dependencies. This method can both be used to generate

\footnotetext{
${ }^{2}$ We used the public domain tool $l p$ solve which is available through FTP at ftp.es.ele.tue.nl.
} 
constraints from a type specification directly or to complete partially filled constraints. For this purpose, the data units for which constraints must be derived have to be specified in Attributed ASN.1. The restriction thereby is that all expressions used to specify the internal dependencies must be linear. We think that for practical protocols this restriction can always be satisfied. By building a prototype tool that implements our approach, we have shown its practical feasibility.

A tool implementing a constraint generation method is very useful in specifying conformance tests as it relieves the test specifier from the task to completely specify all constraints. Furthermore, joining the ideas on external dependencies as presented in [6], test generation algorithms for FSMs [1, 2] and our constraint generation method, allows for the generation of complete tests for all know test generation methods.

Acknowledgements: We like to thank Egbert J. Woerlee who helped us to implement the prototype constraint generation tool and Michel Berkelaar from the Technical University Eindhoven, who let us use his linear programming software. Furthermore, we like to thank Stan P. van de Burgt for his many helpful suggestions and comments to improve this paper.

\section{References}

[1] A.V. Aho, A.T. Dahbura, D. Lee, and M. Umit Uyar. An Optimization Technique for Protocol Conformance Test Generation Based on UIO Sequences and Rural Chinese Postman Tours. In S. Aggarwal and K. Sabnani, editors, Protocol Specification, Testing and Verification: VIII, June 1988.

[2] S.P. van de Burgt. Test Sequence Algorithms and Formal Languages. In Mary E. Kavanaugh, editor, Second Asian Test Symposium, pages 138-146. IEEE Computer Society Press, November 1993.

[3] S.P. van de Burgt and P.A.J. Tilanus. Attributed ASN.1. In Proc. of the 2nd International Conference on Formal Description Techniques, FORTE 89, December 1989. Participants proceedings, also PTT Research technical report Pub 999 RNL/89.

[4] CCITT Recommendations T.431-T.564. Terminal equipment and protocols for telematic services, November 1988. Blue book Volume VII - Fascicle VII.7.

[5] ISO-9646. Conformance testing Methodology and Framework, 1990.

[6] Erik Kwast. Automatic generation for protocol data aspects. In Protocol Specification, Testing, and Verification XII, volume C-8 of IFIP Transactions, pages 211-226. North-Holland, 1992.

[7] Alexander Schrijver. Theory of linear and integer programming. John Wiley \& Sons, 1986.

[8] A.P. Varvitsiotis and G.I. Stassinopoulos. Extending ASN.1 into a full-fledged constraint language in the context of OSI protocol conformance testing. Computer Networks and ISDN systems, 25:1243-1263, 1993. 